\title{
Multiplicity of positive solutions for a critical quasilinear Neumann problem
}

\author{
Aleksandr Enin 1
}

\begin{abstract}
We establish the multiplicity of positive solutions to a quasilinear Neumann problem in expanding balls and hemispheres with critical exponent in the boundary condition.
\end{abstract}

\section{Introduction}

We consider the following problem

$$
\left\{\begin{array}{l}
\Delta_{p} u:=\operatorname{div}\left(|\nabla u|^{p-2} \nabla u\right)=|u|^{p-2} u \quad \text { in } B_{R} \\
|\nabla u|^{p-2}\langle\nabla u ; \mathbf{n}\rangle=|u|^{q-2} u \quad \text { on } S_{R} \\
u>0 \quad \text { in } B_{R}
\end{array}\right.
$$

where $B_{R}$ and $S_{R}$ are the ball and the sphere with radius $R$ respectively in $\mathbb{R}^{n}$. Here $1<p<n$ and $q=p^{* *}=\frac{(n-1) p}{(n-p)}$ is the critical exponent for the trace embedding.

We establish the multiplicity effect for weak solutions to (1). Namely we prove that the number of positive rotationally non-equivalent solutions is unbounded as $R \rightarrow \infty$.

The effect of multiplicity was discovered by Coffman [5] who considered the Dirichlet problem

$$
\left\{\begin{array}{l}
-\Delta_{p} u=|u|^{q-2} u \quad \text { in } \Omega_{R} \\
u=0 \text { on } \partial \Omega_{R} \\
u>0 \quad \text { in } \Omega_{R}
\end{array}\right.
$$

where $\Omega_{R}$ is the annulus $B_{R} \backslash B_{R-1} \subset \mathbb{R}^{n}$ for $n=2$ and $p=2$. The problems (11) and (2) were studied later by many authors for subcritical $q$ (see e.g. [17, 8, 10, 11, 4]). In [20] the multiplicity result was obtained for the Neumann problem

$$
\left\{\begin{array}{l}
-\Delta u+\lambda u=|u|^{p^{*}-2} u \text { in } \Omega, \\
\frac{\partial u}{\partial \nu}=0 \text { on } \partial \Omega \\
u>0 \text { in } \Omega
\end{array}\right.
$$

where $\Omega$ satisfies some symmetry conditions and $p^{*}$ is the critical exponent for the Sobolev trace embedding.

\footnotetext{
${ }^{1}$ Saint Petersburg State University, St. Petersburg, Russia; Saint Petersburg Electrotechnical University "LETI", St. Petersburg, Russia; Aleksandr.Enin@outlook.com
} 
One can easily show that after suitable rescaling solutions of (1) are solutions to the following problem:

$$
\left\{\begin{array}{l}
\Delta_{p} u=\lambda|u|^{p-2} u \quad \text { in } B \\
|\nabla u|^{p-2}\langle\nabla u ; \mathbf{n}\rangle=|u|^{q-2} u \text { on } S \\
u>0 \text { in } B
\end{array}\right.
$$

where $B=B_{1}, S=S_{1}$ and $\lambda(R)=R^{p}$ as $R \rightarrow \infty$.

We look for distinct solutions of the problem (3) by minimizing the functional

$$
I^{\lambda}[u]:=\frac{\|\nabla u\|_{L_{p}(B)}^{p}+\lambda\|u\|_{L_{p}(B)}^{p}}{\|u\|_{L_{q}(S)}^{p}}
$$

on different subsets of $W_{p}^{1}(B)$.

In order to construct solutions to problem (3) let us introduce the following notation:

Definition 1. Let $A \subset S$ and $\varkappa>0$. We denote by $A^{\delta} \varkappa$-neighborhood of a set $A$, i.e.

$$
A^{\varkappa}=\{z \in S \mid \operatorname{dist}(z, A) \leq \delta\}
$$

The following definition was introduced in [4]:

Definition 2. Let $G$ be a closed subgroup of $O(n)$. We call set $A \subset S$ a locally minimal orbital set under the action of $G$ if $A$ is invariant under the action of $G$ and satisfies the following conditions:

- for any $x \in A$ the orbit $G x$ is a discrete set and $m(A):=|G x|$ is independent of $x$.

- there exists $\varkappa>0$ such that for any $y \in A^{\varkappa} \backslash A$ and $x \in A$, we have $|G x|<|G y|$.

We denote as $m(G)$ the number of elements in the minimal orbit of $G$ and $K(n, p)$ stands for the best Sobolev trace constant in half-space defined as

$$
K(n, p)=\inf _{v \in C_{c}^{\infty}\left(\overline{\mathbb{R}_{+}^{n}}\right) \backslash\{0\}} \frac{\|\nabla v\|_{L_{p}\left(\mathbb{R}_{+}^{n}\right)}^{p}}{\|v(\cdot, 0)\|_{L_{q}\left(\mathbb{R}^{n-1}\right)}^{p}} .
$$

The value of $K(n, p)$ is calculated explicitly in [9] for $p=2$ and [13] for arbitrary $p$.

We consider local minimizers of functional (4) on sets

$$
X_{G}(A, \beta)=\left\{u \in W_{p}^{1}(B) \mid u(g x) \equiv u(x) \forall g \in G,\|u\|_{L_{q}(S)}=1,\|u\|_{L_{q}\left(A^{\varkappa}\right)}^{q} \geq 1-\beta\right\},
$$

where $G$ is some closed subgroup of $O(n), A$ is a locally minimal orbital set and $\beta$ is some small parameter that we will choose later. We denote $X_{G}(A, \beta)$ by $X$ if it does not lead to confusion.

The structure of the paper is as follows. In Section 2 we prove some auxiliary lemmas and in Section 3 we establish main multiplicity results. 


\section{Auxiliary lemmas}

The following fact is well known and will be given here without a proof.

Proposition 1. The functional $I^{\lambda}[u]$ is Gateaux differentiable and for any $h \in W_{p}^{1}(B)$

$$
\begin{aligned}
D I^{\lambda}[u](h) & =p \int_{B}|\nabla u|^{p-2} \nabla u \cdot \nabla h d x \frac{1}{\|u\|_{L_{q}(S)}^{p}}-p \int_{B}|\nabla u|^{p} d x \int_{S}|u|^{q-2} u h d S \frac{1}{\|u\|_{L_{q}(S)}^{p+q}} \\
& -p \lambda \int_{B}|u|^{p} d x \int_{S}|u|^{q-2} u h d S \frac{1}{\|u\|_{L_{q}(S)}^{p+q}}+p \lambda \int_{B}|u|^{p-2} u h d x \frac{1}{\|u\|_{L_{q}(S)}^{p}} .
\end{aligned}
$$

Lemma 1. Let $u_{j}^{\lambda} \in W_{p}^{1}(B)$ be a bounded Palais-Smale sequence for $I^{\lambda}$ at the level $c>0$. Then there is $u_{0}^{\lambda} \in W_{p}^{1}(B)$ such that up to subsequence $u_{j}^{\lambda} \rightarrow u_{0}^{\lambda}$ and

$$
\begin{array}{r}
\left|\nabla u_{j}^{\lambda}\right|^{p} d x \rightarrow \mu \geq\left|\nabla u_{0}^{\lambda}\right|^{p} d x+\sum_{k} \mu_{k} \delta\left(x-x_{k}\right), \\
\left|u_{j}^{\lambda}\right|^{q} d S \rightarrow \nu=\left|u_{0}^{\lambda}\right|^{q} d S+\sum_{k} \nu_{k} \delta\left(x-x_{k}\right),
\end{array}
$$

where $\delta\left(x-x_{k}\right)$ are delta measures at some points $x_{k}$ in $S$ and $\mu_{k} \geq K(n, p) \nu_{k}^{\frac{p}{q}}$. Furthermore, either $\nu_{k}=0$ or $\nu_{k} \geq\left(c^{-1} \cdot K(n, p)\right)^{\frac{q}{q-p}} \nu(S)$.

Proof. Since $\left\{u_{j}^{\lambda}\right\}$ is bounded in $W_{p}^{1}(B)$, the relations (5) and (6) follow by the Lions concentration-compactness principle [12]. Since $I^{\lambda}$ is homogeneous we can assume without loss of generality that $\left\|u_{j}^{\lambda}\right\|_{L_{q}(S)}=1$ and $\nu(S)=1$. Next we use the argument from [6, 1]: Let us fix $x_{k}$ from (5) and ([6). We choose $\varphi \in C_{c}^{\infty}\left(\mathbb{R}^{n}\right)$ such that

$$
\varphi=1 \text { in } B\left(x_{k}, \varepsilon\right), \quad \varphi=0 \text { in } \mathbb{R}^{n} \backslash B\left(x_{k}, 2 \varepsilon\right), \quad|\nabla \varphi| \leq \frac{C}{\varepsilon} .
$$

Since $D I^{\lambda}\left[u_{j}^{\lambda}\right] \rightarrow 0$ we obtain

$$
\lim _{j \rightarrow \infty}<D I^{\lambda}\left[u_{j}^{\lambda}\right], \varphi u_{j}^{\lambda}>=0
$$

Then

$$
\lim _{j \rightarrow \infty} \int_{B}\left|\nabla u_{j}^{\lambda}\right|^{p-2} \nabla u_{j}^{\lambda} \cdot \nabla \varphi_{j} u_{j}^{\lambda} d x=c \int_{S} \varphi d \nu-\int_{B} \varphi d \mu-\lambda \int_{B}\left|u_{0}^{\lambda}\right|^{p} \varphi d x .
$$

One can estimate the left hand side as follows:

$$
\begin{aligned}
0 & \leq\left.\left|\lim _{j \rightarrow \infty} \int_{B}\right| \nabla u_{j}^{\lambda}\right|^{p-2} \nabla u_{j}^{\lambda} \cdot \nabla \varphi u_{j}^{\lambda} d x \mid \\
& \leq \lim _{j \rightarrow \infty}\left(\int_{B}\left|\nabla u_{j}^{\lambda}\right|^{p} d x\right)^{\frac{p-1}{p}}\left(\int_{B}|\nabla \varphi|^{p}\left|u_{j}^{\lambda}\right|^{p} d x\right)^{\frac{1}{p}} \\
& \leq C\left(\int_{B\left(x_{k}, 2 \varepsilon\right)}|\nabla \varphi|^{p}\left|u_{0}^{\lambda}\right|^{p} d x\right)^{\frac{1}{p}} \\
& \leq C\left(\int_{B\left(x_{k}, 2 \varepsilon\right)}|\nabla \varphi|^{n} d x\right)^{\frac{1}{n}}\left(\int_{B\left(x_{k}, 2 \varepsilon\right)}\left|u_{0}^{\lambda}\right|^{\frac{n p}{n-p}} d x\right)^{\frac{n-p}{p n}} \\
& \leq C\left(\int_{B\left(x_{k}, 2 \varepsilon\right)}\left|u_{0}^{\lambda}\right|^{\frac{n p}{n-p}} d x\right)^{\frac{n-p}{p n}} \rightarrow 0 \text { as } \varepsilon \rightarrow 0 .
\end{aligned}
$$


Taking the limit in (7) we get $\nu_{k}=c^{-1} \mu_{k} \geq c^{-1} K(n, p) \nu_{k}^{\frac{p}{q}}$. This means either $\nu_{k} \geq\left(c^{-1} K(n, p)\right)^{\frac{q}{q-p}}$ or $\nu_{k}=0$.

Lemma 2. Let $u^{\lambda} \in X$ be a sequence such that $I^{\lambda}\left[u^{\lambda}\right] \leq K(n, p) m(A)^{1-\frac{p}{q}}$. Then there is a $\beta_{0}>0$ such that for any $\beta \leq \beta_{0}$ there is $x_{0} \in S$ such that we have up to subsequence the following weak convergence in the sense of measures as $\lambda \rightarrow \infty$ :

$$
\left|u^{\lambda}\right|^{q} d S \rightarrow \sum_{x_{k} \in G x_{0}} \frac{1}{m(A)} \delta\left(x-x_{k}\right) .
$$

Proof. Since $\left\|u^{\lambda}\right\|_{W_{p}^{1}}^{p} \leq I^{\lambda}\left[u^{\lambda}\right] \leq K(n, p) m(A)^{1-\frac{p}{q}}$ by the Lions concentrationcompactness principle we get

$$
\begin{array}{r}
\left|\nabla u^{\lambda}\right|^{p} d x \rightarrow \mu \geq\left|\nabla u_{0}\right|^{p} d x+K(n, p) \sum_{k} \nu_{k}^{\frac{p}{q}} \delta\left(x-x_{k}\right), \\
\left|u^{\lambda}\right|^{q} d S \neg \nu=\left|u_{0}\right|^{q} d S+\sum_{k} \nu_{k} \delta\left(x-x_{k}\right),
\end{array}
$$

where $\delta\left(x-x_{k}\right)$ are delta measures at some points $x_{k}$ in $S$.

Since $\lambda\left\|u^{\lambda}\right\|_{L_{p}(B)}^{p}$ is uniformly bounded, we have $u^{\lambda} \rightarrow 0$ in $L_{p}(B)$ so $u_{0}=0$. Combining the above with the fact that $u^{\lambda}$ are invariant with respect to $G$ we get:

$$
\begin{aligned}
\lim _{\lambda \rightarrow \infty} I^{\lambda}\left[u^{\lambda}\right]=\mu(B) \geq K(n, p) \sum_{k} \nu_{k}^{\frac{p}{q}} & =K(n, p) \sum_{j}\left|G x_{j}\right|\left(\frac{\tilde{\nu_{j}}}{\left|G x_{j}\right|}\right)^{\frac{p}{q}} \\
& =K(n, p) \sum_{j}\left|G x_{j}\right|^{1-\frac{p}{q}}{\tilde{\nu_{j}}}^{\frac{p}{q}} .
\end{aligned}
$$

Here $j$ goes over different classes of equivalence of $x_{k}$, and $\tilde{\nu}_{j}=\left|G x_{j}\right| \nu_{j}$ is a total contribution of that class to $\nu(\partial \Omega)$. The second equality is due to the fact that $u^{\lambda}$ are $G$-invariant, so for every $x_{k}$ there are $\left|G x_{k}\right| \delta$-functions with the same coefficient.

Since $p<q$ we have $a^{\frac{p}{q}}+b^{\frac{p}{q}}>(a+b)^{\frac{p}{q}}$, for any $a>0, b>0$. Recalling that $A$ is a locally minimal orbital set we can write

$$
\begin{aligned}
\mu(B) & \geq K(n, p) m(A)^{1-\frac{p}{q}} \sum_{j: x_{j} \in A} \tilde{\nu}_{j}^{\frac{p}{q}}+K(n, p) \sum_{i: x_{i} \notin A}\left|G x_{i}\right|^{1-\frac{p}{q}} \tilde{\nu}_{i}^{\frac{p}{q}} \geq \\
& \geq K(n, p)\left(m(A)^{1-\frac{p}{q}} \alpha^{\frac{p}{q}}+m(G)^{1-\frac{p}{q}}(1-\alpha)^{\frac{p}{q}}\right),
\end{aligned}
$$

where $1-\beta \leq \alpha \leq 1$ (we recall that $m(G)$ is the number of elements in the minimal orbit of $G$ ).

It's easy to see that the right hand side of (10) is a concave function of $\alpha$. That means that if $\beta$ is small enough, then the right hand side is a decreasing function, which achieves it's minimum of $K(n, p) m(A)^{1-\frac{p}{q}}$ at $\alpha=1$.

Since by assumption $\mu(B)=\lim _{\lambda \rightarrow \infty} I^{\lambda}\left[u^{\lambda}\right] \leq K(n, p) m(A)^{1-\frac{p}{q}}$ we conclude that $\alpha=1$. Recalling that for $u \in X\|u\|_{L_{q}(S)}=1$ we get (8) $)$.

From now on we always assume that $\lambda$ is fixed and whenever there is a limit it is taken over $j \rightarrow \infty$ unless specified otherwise. 
Lemma 3. The minimum of $I^{\lambda}$ on $X$ is attained if $\lambda$ is large enough and

$$
\inf _{u \in X} I^{\lambda}[u]<K(n, p) m(A)^{1-\frac{p}{q}}
$$

Proof. The Ekeland's variational principle [7] provides the existence of a minimizing sequence $u_{j}^{\lambda} \in X$ such that $I^{\prime}\left[u_{j}^{\lambda}\right] \rightarrow 0$. Since $u_{j}^{\lambda}$ is a Palais-Smale sequence at the level $\inf _{u \in X} I^{\lambda}[u]<K(n, p) m(A)^{1-\frac{p}{q}}$, Lemma 1 gives the estimate on any non-zero $\nu_{k}$ in ([6]):

$$
\nu_{k}>m(A)^{-\left(1-\frac{p}{q}\right) \frac{q}{q-p}}=\frac{1}{m(A)} .
$$

Suppose that there is a $\delta$-function outside of $A$. From (8) follows that for large $\lambda$ almost all of $\nu(S)$ mass is concentrated in a $\varkappa$-neighbourhood of $A$, and according to (11) there are no $\delta$-functions outside of that neighbourhood.

Let us suppose that there is a $\delta$-function at $x_{k} \in A^{\varkappa}$ with weight $\nu_{k}$. Since $A$ is a locally minimal orbital set, we know that $\left|G x_{k}\right| \geq m(A)$. Now from (9) and (11) we derive

$$
\lim _{j \rightarrow \infty} I^{\lambda}\left[u_{j}^{\lambda}\right] \geq K(n, p)\left|G x_{k}\right|\left(\frac{1}{m(A)}\right)^{\frac{p}{q}}=K(n, p) m(A)^{1-\frac{p}{q}}
$$

which is a contradiction.

From that follows that for $u_{0}^{\lambda}$ in (18) $\left\|u_{0}^{\lambda}\right\|_{L_{q}(S)}=\left\|u_{j}^{\lambda}\right\|_{L_{q}(S)}=1$. It is well known, that weak convergence and convergence of norms implies strong convergence in uniformly convex Banach space (e.g. [3, Proposition 3.32]), and that completes our proof. That way $u_{0}^{\lambda} \in X$ and $I^{\lambda}\left[u_{0}^{\lambda}\right]$ attains minimal value.

\section{Main results}

Lemma 4. Let $G=H \times O(n-k)$ where $H$ is a finite subgroup of $O(k)$ and $A \subset \mathbb{R}^{k}$ is a minimal orbital set under the action of $H$.

Then for any fixed $\beta, \lambda$ large enough and $p \leq \frac{n+1}{2}$ we have

$$
\inf _{u \in X} I^{\lambda}\left[u^{\lambda}\right]<K(n, p) m(A)^{1-\frac{p}{q}} .
$$

Proof. Let $x_{0} \in G x_{0}$ be a point in $A \times\{0\}$. As was shown in [14] (see also [2]) there is a function $u_{R}$ in $W_{p}^{1}\left(B_{R}\right)$ supported in a small ball around $R x_{0}$ and axially symmetric with respect to the axis $O x_{0}$, such that $\left\|u_{R}\right\|_{W_{p}^{1}\left(B_{R}\right)}^{p}<K(n, p)\|u\|_{L_{q}\left(S_{R}\right)}^{p}$.

Now we construct the function

$$
v_{R}(x)=\sum_{g \in H} u_{R}(g x)
$$

It is easy to see that $v_{R}$ is $G$-invariant and

$$
\frac{\left\|v_{R}\right\|_{W_{p}^{1}\left(B_{R}\right)}^{p}}{\left\|v_{R}\right\|_{L_{q}\left(S_{R}\right)}^{p}}=m(A)^{1-\frac{p}{q}} \frac{\left\|u_{R}\right\|_{W_{p}^{1}\left(B_{R}\right)}^{p}}{\left\|u_{R}\right\|_{L_{q}\left(S_{R}\right)}^{p}}<K(n, p) m(A)^{1-\frac{p}{q}} .
$$

By rescaling we obtain (13). 
Theorem 1. Let $p \leq \frac{n+1}{2}$ and let $G$ be as in Lemma 4. Suppose that $A \subset \mathbb{R}^{k}$ is some locally minimal orbital set of $H$. Then there is $\lambda_{0}>0$ such that for any $\lambda>\lambda_{0}$ there is a $G$-invariant solution of problem (3) such that it concentrates at $\left|G x_{0}\right|$ points in the $G x_{0}$ for some $x_{0} \in A \times\{0\}$, i.e.

$$
\frac{\left|u^{\lambda}\right|^{q}}{\left\|u^{\lambda}\right\|_{L_{q}(S)}} \rightarrow \sum_{k=1}^{\left|G\left(x_{0}\right)\right|} \frac{1}{\left|G\left(x_{0}\right)\right|} \delta\left(x-x_{k}\right) \quad \text { as } \quad \lambda \rightarrow \infty .
$$

Proof. According to Lemmas 4 and 3 there is a minimizer $u \in X$ such that it is concentrated around $m(A)$ points of $A \times\{0\}$. Lemma 2 implies that if $\lambda$ is large enough the constraint $\|u\|_{L_{q}\left(A^{\delta}\right)}^{q}>1-\beta$ is non-active and does not produce a Lagrange multiplier. Since $I^{\lambda}[u]=I^{\lambda}[|u|]$ we can assume that $u$ is non-negative. Since $u$ is a local minimizer, we get for $\mu=I^{\lambda}[u]$ (see Proposition [1):

$$
\int_{B}|\nabla u|^{p-2} \nabla u \cdot \nabla h d x+\lambda \int_{B}|u|^{p-2} u h d x-\mu \int_{S}|u|^{q-2} u h d S=0 \quad \forall h \in L_{G},
$$

where

$$
L_{G}=\left\{h \in W_{p}^{1}(B) \mid h(g x)=h(x) \forall g \in G\right\} .
$$

Due to the principle of symmetric criticality [16] $u$ is a solution to the problem

$$
\left\{\begin{array}{l}
\Delta_{p} u:=\lambda|u|^{p-2} u \quad \text { in } B \\
|\nabla u|^{p-2}\langle\nabla u ; \mathbf{n}\rangle=\mu|u|^{q-2} u \quad \text { on } S,
\end{array}\right.
$$

Since $u \geq 0$ in $B$ we can apply the Harnack inequality (see [19], [18]) and get the positivity of our solution. Since the boundary condition is not homogeneous, it's easy to show that $\mu^{\frac{1}{p-q}} u$ is a solution for problem (3).

Theorem 2. For any $N>0$ there is $\lambda_{0}>0$ such that for every $\lambda>\lambda_{0}$ problem (3) has at at least $N$ distinct solutions.

Proof. Let us look at the following decomposition of $\mathbb{R}^{n}$ :

$$
\mathbb{R}^{n}=\left(\mathbb{R}^{2}\right)^{l} \times \mathbb{R}^{m} .
$$

Here $l \geq 1, m \geq 0$. We denote variables in $\mathbb{R}^{n}$ by $x$, in $\mathbb{R}^{2}$ by $y$ and in $\mathbb{R}^{m}$ by $z$. This way,

$$
x=\left(y_{1}, y_{2}, \ldots, y_{l} ; z\right) .
$$

We introduce the group $G_{k, l}=H_{k, l} \times O(m)$ where $H_{k, l}$ is generated by rotations of every $y_{i}$ by $\frac{2 \pi}{k}$ and by transpositions of $y_{i}$ and $y_{j}$ for every $i$ and $j$.

Let $A$ be a globally minimal orbital set for the action of $H_{k, l}$. One can easily check that $A \times\{0\}$ is a locally minimal orbital set for $G_{k, l}$.

Now we show that for $l \geq 1$ and $k>2$ the minimizers will be non-equivalent. In order to do that we analyse minimal orbits of $H_{k, l}$. The simple calculation yields that a minimal orbit would be of a point $(y, 0, \ldots, 0) \in \mathbb{R}^{2 l}$ where $y \in \mathbb{R}^{2}$ and it consists of $k \cdot l$ points. Knowing 
the structure of the minimal orbits we can deduce that minimizers would be different for different pairs of $(k, l)$ and $\left(k^{\prime}, l^{\prime}\right)$.

Now we consider an analogue of the problem (3) in an $n$-dimensional hemisphere.

To prove the multiplicity result we only need to modify lemma 4 by using the existence result from [15].

Lemma 5. Let $n \geq 5$ and let $B$ be an $n$-dimensional hemisphere. Let $G=H \times O(n-k)$ where $H$ is a finite subgroup of $O(k)$ such that $A$ is a minimal orbital set under the action of $H \times\{0\}$.

Then for any fixed $\beta, \lambda$ large enough and $2<p \leq \frac{n+2}{3}$ we have

$$
\inf _{u \in X} I^{\lambda}\left[u^{\lambda}\right]<K(n, p) m(A)^{1-\frac{p}{q}} .
$$

Repeating the previous arguments we get the following theorem:

Theorem 3. Let $n \geq 5$, and let $B$ be an $n$-dimensional hemisphere, $2<p \leq \frac{n+2}{3}$. Then for any $N>0$ there is a $\lambda_{0}>0$ such that for any $\lambda>\lambda_{0}$ problem (3) has at least $N$ rotationally non-equivalent solutions.

\section{Acknowledgements}

I am grateful to A.I. Nazarov for stimulating discussions.

The work has been supported by RFBR №14-01-00534.

A part of this paper was written during the visit of author to University of Cologne, supported by joint program of DAAD and St. Petersburg State University "Dmitrij Mendeleev". I am grateful to professor Bernd Kawohl for the hospitality during this visit.

\section{References}

[1] Bonder, J. F., Rossi J. D. "On the existence of extremals for the Sobolev trace embedding theorem with critical exponent." Bulletin of the London Mathematical Society 37.1 (2005): 119-125.

[2] Bonder, J. F., Saintier N. "Estimates for the Sobolev trace constant with critical exponent and applications." Annali di Matematica Pura ed Applicata 187.4 (2008): 683-704.

[3] Brezis, H. Functional analysis, Sobolev spaces and partial differential equations. Universitext. Springer, New York, (2011).

[4] Byeon, J. "Existence of many nonequivalent nonradial positive solutions of semilinear elliptic equations on three-dimensional annuli." Journal of differential equations 136.1 (1997): 136-165.

[5] Coffman, C. V. "A non-linear boundary value problem with many positive solutions." Journal of Differential Equations 54.3 (1984): 429-437. 
[6] Demengel, F., Nazaret B. "On some nonlinear partial differential equations involving the p-Laplacian and critical Sobolev trace maps." Asymptotic Analysis 23.2 (2000): $135-156$.

[7] Ekeland, I. "On the variational principle." Journal of Mathematical Analysis and Applications 47.2 (1974): 324-353.

[8] Enin A. I., Nazarov A. I., Multiplicity of solutions to the quasilinear Neumann problem in the 3-dimensional case, J. Math. Sci. 207.2 (2015): 206-217.

[9] Escobar, J. F. "Sharp constant in a Sobolev trace inequality." Indiana University Mathematics Journal 37, no. 3 (1988): 687-698.

[10] Kolonitskii, S. B. "Multiplicity of solutions of the Dirichlet problem for an equation with the p-Laplacian in a three-dimensional spherical layer." St. Petersburg Mathematical Journal 22.3 (2011): 485-495.

[11] Li, Y. Y. "Existence of many positive solutions of semilinear elliptic equations on annulus." Journal of Differential Equations 83.2 (1990): 348-367.

[12] Lions, P. L. "The concentration-compactness principle in the calculus of variations. The limit case, part 2." Rev. Mat. Iberoamericana 1.2 (1985): 45-121.

[13] Nazaret, B. "Best constant in Sobolev trace inequalities on the half-space." Nonlinear Analysis: Theory, Methods \& Applications 65, no. 10 (2006): 1977-1985.

[14] Nazarov, A. I., Reznikov A. B. "On the existence of an extremal function in critical Sobolev trace embedding theorem." Journal of Functional Analysis 258.11 (2010): 39063921.

[15] Nazarov, A. I., Reznikov A. B. "Attainability of infima in the critical Sobolev trace embedding theorem on manifolds." Nonlinear Partial Differential Equations and Related Topics: Dedicated to Nina N. Uraltseva 64 (2010): 197-210.

[16] Palais, R. S. "The principle of symmetric criticality." Communications in Mathematical Physics 69.1 (1979): 19-30.

[17] Scheglova, A. P. "Multiplicity of solutions to a boundary-value problem with nonlinear Neumann condition." Journal of Mathematical Sciences 128.5 (2005): 3306-3333.

[18] Serrin, J. "On the Harnack inequality for linear elliptic equations." Journal d'Analyse Mathématique 4.1 (1954): 292-308.

[19] Trudinger, N. S. "On Harnack type inequalities and their application to quasilinear elliptic equations." Communications on Pure and Applied Mathematics 20.4 (1967): 721-747.

[20] Wang, Z. Q. "Construction of multi-peaked solutions for a nonlinear Neumann problem with critical exponent in symmetric domains." Nonlinear Analysis: Theory, Methods \& Applications 27.11 (1996): 1281-1306. 